\title{
QoS Support for Multi-user Sessions in IP-based Next Generation Networks
}

\author{
Eduardo Cerqueira - Luis Veloso - Augusto Neto • \\ Marilia Curado • Edmundo Monteiro • Paulo Mendes
}

Published online: 17 June 2008

(C) Springer Science + Business Media, LLC 2008

\begin{abstract}
A combined control of multimedia quality level, mobility and allocation of network resources is essential for the success of next generation mobile networks. In this context, this article presents the Multi-user Session Control (MUSC) solution to control the quality level of multimedia sessions shared by multiple-users, providing Quality of Service (QoS) mapping and QoS adaptation for those sessions over heterogeneous and mobile networks. MUSC uses the self-organized principle to coordinate QoS mapping and QoS adaptation mechanisms with mobility and resource allocation controllers, allowing the adaptation of a session to the current network conditions and the dynamic selection of the suitable network service class to map the session. MUSC minimizes the blocking probability, opti-
\end{abstract}

Work developed under the affiliation of NTT DoCoMo Euro-Labs, Munich, Germany

E. Cerqueira $(\bowtie) \cdot$ L. Veloso $\cdot$ A. Neto $\cdot$ M. Curado $\cdot$ E. Monteiro Department of Informatics Engineering, University of Coimbra,

Polo II, Pinhal de Marrocos,

3030029 Coimbra, Portugal

e-mail: ecoelho@dei.uc.pt

L. Veloso

e-mail: lmveloso@dei.uc.pt

A. Neto

e-mail: augusto@dei.uc.pt

M. Curado

e-mail: marilia@dei.uc.pt

E. Monteiro

e-mail: edmundo@dei.uc.pt

P. Mendes

INESC Porto,

Rua Dr. Roberto Frias, 378,

4200465 Porto, Portugal

e-mail: pmendes@inescporto.pt mizes the usage of network resources and keeps sessions with an acceptable quality of experience. MUSC was evaluated in a simulation and in an experimental environment to analyze its convergence time, percentage of session blocking as well as delay, throughput, Peak Signal-to-Noise Ratio (PSNR), Mean Option Scores (MOS) and Structural Similarity Index (SSIM) of sessions in QoS-aware mobile scenarios.

Keywords multi-user session - quality of service - mobility · heterogeneous networks

\section{Introduction}

The Quality of Service (QoS) control is an important research topic in fixed and mobile networks, due to the use of different network service classes, the dynamic behaviour of wired and wireless resources, and the emergent market of real-time communications. Examples of these communication sessions are IPTV, video streaming, seismic activity report and other kinds of multimedia as well as sensor networks applications. Since the content is simultaneously destined to multiple users, this type of sessions are called multi-user sessions.

Well-know CODECs, such as Moving Picture Experts Group version 4 (MPEG-4) can also support scalable multiuser sessions. Each scalable session can be composed by a set of flows, with well-defined priorities, rates and QoS requirements. The importance of each flow must be used to adapt the overall quality of the session according to the type of service classes supported by different networks. This scheme allows the network to be independent from the encoders, which does not happen with transcoding approaches [1]. Figure 1 illustrates a generic example of 


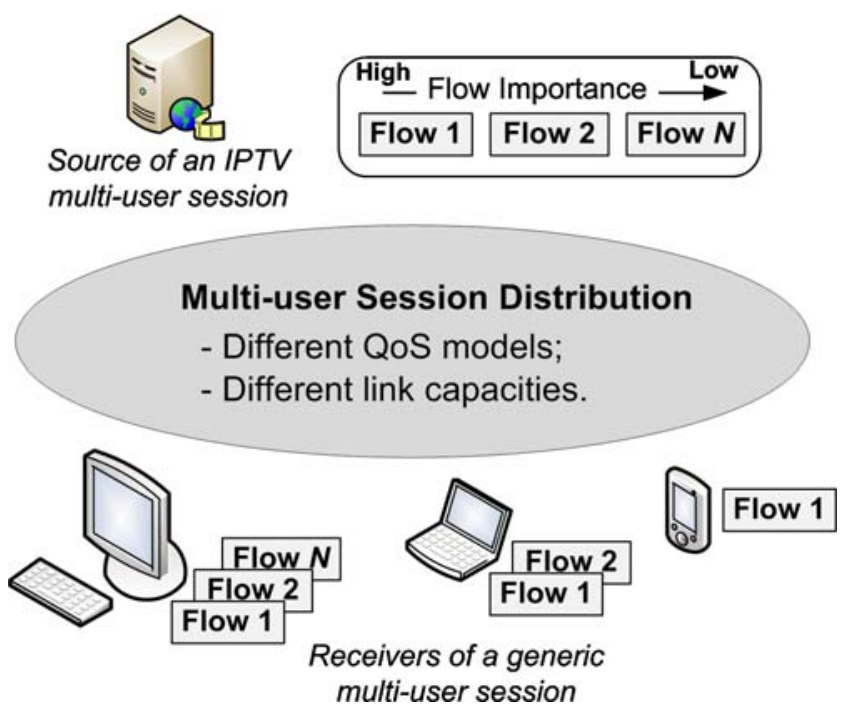

Figure 1 Example of a multi-user session definition and distribution environment

an IPTV session distribution over networks with different QoS models and link capacities, where a multi-user session is subscribed by different devices and distributed according to the importance of each flow (from the highest to the lowest importance flow).

The end-to-end multi-user session distribution over heterogeneous networks must be performed independently of the QoS models supported along the session path. For instance, Differentiated Service (DiffServ) or Integrated Service (IntServ) can be implemented to provide QoS assurance for sessions in wired links, while IEEE 802.16 (IEEE $802.16-2004$ or IEEE $802.16 \mathrm{e}-2005$ ) or IEEE 802.11 e can be used in wireless links.

Network services supported by class-based QoS models (e.g., DiffServ and IEEE 802.11e) offer different forwarding behaviours for packets. Hence, the definition of each QoS class is based on a set of performance metrics such as, committed bandwidth, tolerance to loss, delay and jitter. In each network along the end-to-end heterogeneous session path, flows of multi-user sessions with similar QoS requirements must be mapped into the appropriated service class. However, static approaches of QoS mapping between session requirements and network service classes or even guidelines for IP QoS mapping [2] alone, are not sufficient to assure the quality level of sessions. This is mainly due to the emergence of sessions with new QoS requirements and QoS models with different configurations and classes of service. In addition, the network service classes can be configured in different manners (using different QoS performance metrics) in order to satisfy the business scheme of each network operator.

Furthermore, due to the existence of links with distinct capacities, oscillation of network capabilities and unpre- dictable re-routing events (e.g., caused by handovers or link failures), QoS mapping operations must be accomplished together with QoS adaptation support. The latter avoids the session blocking and keeps sessions with an acceptable quality level, independently of the movement of users or topology changes. For instance, in a congestion period, a QoS adaptation mechanism must be used to adapt the session to the current network conditions, by requesting the re-mapping of the session to a different class or controlling the quality level of the scalable session by dropping or adding low priority flows.

Summing up, the end-to-end quality level control of multi-user sessions must be done independently of QoS models, capability of the links and re-routing events. Moreover, this control must be provided for unicast or multicast sessions following a sender-initiated approach to assure the distribution of QoS-aware sessions in environments with asymmetric routing. In mobility situations, the QoS support for ongoing multi-user sessions must be accomplished independently of mobility schemes, such as the bi-directional tunnelling based on the Mobile IP (MIP) [3], the solutions based on the Session Initiation Protocol (SIP) [4] or the Multicast Remote-Subscription [5] approaches.

Our previous work describes the benefits of combining QoS mapping and adaptation mechanisms in the session quality level, by implementing a QoS-aware multicast testbed to measure the throughput of a session when the system is configured to adapt sessions by dropping and adding low priority flows [6]. MUSC capabilities to control the quality level of multi-user sessions were also analyzed in a simulation environment, by verifying throughput and one-way-delay of sessions, when several QoS mapping and adaptation methods are used [7]. This article extends our previous work by presenting the effective gain of the combination of QoS mapping, QoS adaptation, mobility and resource allocation controllers in a simulation and in an experimental environment. A selective QoS adaptation scheme is also introduced in order to minimize the number of blocked sessions, while keeping current sessions with an acceptable quality level. Additionally, this article evaluates QoS-related parameters, such as throughput, one-way delay and Round-Trip Time (RTT) delay and Quality of Experience (QoE) parameters of a session, such as Peak Signal-to-Noise Ratio (PSNR), Mean Option Scores (MOS) and Structural Similarity Index (SSIM).

The remainder of this paper is structured as follows. Section 2 presents the related work. The MUSC QoS control proposal is described in Section 3. Examples of MUSC functionality are depicted in Section 4. Performance evaluations are presented in Section 5. Conclusions and future work are shown in Section 6. 


\section{Related work}

The static mapping of sessions across IntServ and DiffServ QoS models is addressed by existing approaches [8]. However, these proposals are dependent of the underlying QoS model. Another static solution is focused on the mapping between Universal Mobile Telecommunications System (UMTS) QoS classes into IP QoS classes [9] following the guideline provided by the ITU-T Recommendation Y.1541. In addition, several guideline-based QoS mapping solutions only control the mapping of sessions from DiffServ or IntServ models to IEEE 802.16 QoS model [10]. Other solutions require the installation of proprietary modules in the end-hosts [11] or the use of a centralized approach [12] to perform the session QoS mapping among networks with different QoS models, reducing the system flexibility and scalability. Moreover, these QoS mapping proposals assume that all neighbouring networks configure their classes with the same QoS performance metrics.

A different QoS mapping solution is introduced by Schantz et al. [13] to control the quality level of sessions in DiffServ-aware home networks. Based on the congestion notification sent by resource allocation controllers, receivers may request the mapping of the session into a less important class. If the session is misplaced and the chosen class cannot accommodate the bandwidth required for the session, the resource allocation controller notifies again the receiver to perform a new QoS mapping or to finish the session. Although not analysed by the author, it seems that this approach leads to high blocking probability, since no further options are given to receivers in order to obtain resources for the desired session. Additionally, this proposal requires changes in all terminals, making its deployment more difficult as well as a constant interaction of non-expert users in the mapping process.

A proposal to control the quality level of sessions based on QoS mapping is proposed by Rajan et al. [14]. This approach defines four DiffServ-based classes with different priorities, and the end-user selects manually one class to be used for each session. If the available resources in the selected class are not enough to satisfy the bandwidth required for the session, the session is rejected or remapped into the best effort class. The adaptation process depends on the manual selection by users, which reduces the system flexibility. In addition, this proposal does not recover the session full quality when resources assigned for the preferred service class become available again.

QoS adaptation control mechanisms are used to adjust the session quality level to the current network conditions. Existing receiver-based solutions require the implementation of proprietary modules in the end-hosts to join or leave flows of multicast sessions based on notifications about the network conditions [15]. Transcoder-based adaptation proposals need network devices to adapt the content coding (re-coding) to the available bandwidth [16], making the network deployment dependent from multimedia encoders. In addition to the previous receiver and transcoder-based approaches, sender-based schemes perform poorly in a heterogeneous multi-user environment [17], because a single transmission rate cannot be used to satisfy the requirements of heterogeneous mobile devices and networks.

The analysis of related work has shown that most QoS mapping proposals use a static guideline scheme to map the sessions without taking into account the current bandwidth of the service classes. In addition, existing mapping schemes were developed to be used in networks with specific QoS models or need the implementation of proprietary modules in mobile devices. Regarding QoS adaptation, the analyzed approaches present the drawback of requiring changes in the end-hosts, the installation of devices to modify the content coding within the network or do not assure the full quality level of sessions when the network resources become available again. To overcome the identified limitations and to keep sessions with an acceptable quality level, while minimizing the session blocking probability, the MUSC QoS control solution is proposed. This approach is being developed in the $Q o S$ for Multi-user Mobile Multimedia (Q3M) project [18, 19].

\section{MUSC QoS control}

MUSC provides a QoS control solution for multi-user sessions over heterogeneous wired and wireless networks. This approach does not require changes in mobile devices, shields end-user and network internals from the details of the QoS infrastructures and assures an acceptable quality level to sessions, even during handover or failure. The MUSC functionalities are implemented by MUSC agents, where a signalling protocol, called MUSC Protocol (MUSC-P) [20], is used to coordinate QoS mapping and QoS adaptation controllers with other agents along the endto-end session path. MUSC agents can be configured in a centralized or decentralized manner. Centralized agents control enforcement points in edge network devices. Decentralized MUSC agents (default approach) are collocated in edge elements.

The MUSC approach assumes that each multi-user session is described in a Session Object (SOBJ) that is identified by a unique session identifier as proposed in the Next Steps in Signalling (NSIS) framework [21]. A multiuser session can be composed by a set of flows, whose QoS parameters are described based on the QSPEC object [22]. Each QSPEC object includes the priority of each flow, 
performance parameters (e.g., bit rate, tolerance to loss, delay and jitter) and traffic metrics (e.g., packet size). These values can be quantitative (e.g., $\mathrm{ms}$ or $\mathrm{Mb} / \mathrm{s}$ ) or qualitative (e.g., low, medium or high). Besides the QoS information collected in the SOBJ, and exchanged between MUSC agents, MUSC receives information regarding the network classes (quantitative/qualitative) from the resource allocation controller, including the available bandwidth and performance parameters.

The MUSC approach is based on a modular integration of session control, resource allocation and mobility control functions to facilitate its deployment as well as the inclusion of existing and emerging solutions. With this goal in mind, a set of open interfaces is defined by MUSC. Figure 2 depicts an overview of the interaction between MUSC, resource allocation and mobility controllers and the interfaces used for the communication between these controllers and existing standards such as, SIP, Session Description Protocol (SDP) [23], Protocol Independent Multicast for Source Specific Multicast (PIM-SSM) [24], Open Shortest Path First (OSPF) [25], Border Gateway Protocol (BGP) [26], MIP, DiffServ elements and Layer 2 wireless controllers.

The MUSC $\leftrightarrow$ SIP/SDP interface allows users to access or leave a multi-user session. SIP is a well-known signalling protocol, designed to be independent of the underlying transport and networks layers, that is widely adopted by vendors, operators and clients to control sessions in the Internet. Upon receiving the session announcement, applications compose a SIP/SDP message to join announced sessions. Receivers use SIP/SDP to request access to a session by passing its SOBJ (described in the SDP) to a SIP proxy, which redirects the request to the edge agent controlling the access point used by the receiver (it may be done after an authentication control). In this edge agent, MUSC processes the collected SOBJ. Afterwards, MUSC coordinates with other edge agents, by using MUSC-P, the quality level to be assigned to the session on the path from its source.

The MUSC $\leftrightarrow$ Resource Allocation Control interface aims to provide end-to-end QoS support of sessions over heterogeneous networks. MUSC triggers the resource allocation controller to retrieve information about the QoS characteristic of the classes inside or between networks and to request the allocation of each flow of a session into a class. MUSC also triggers the resource allocation controller to release the resources of unsubscribed sessions.

The MUSC $\leftrightarrow$ Mobility Control interface allows QoS control for ongoing sessions inside and between networks. By interacting with seamless handover controllers, such as Seamless Mobility of Users for Media Distribution Services (SEMUD) [27], MUSC controls the setup of QoS-aware sessions in advance. Another example is the communication between MUSC and hard handover schemes, such as MIP-alike controllers (MIPv4 or MIPv6), which allows MUSC to control the quality level of an ongoing session also along the path from the Home Agent (HA) to the mobile device attached to a new access-agent and the release of resources on the old path. The interaction between MUSC and MIP-alike controllers aims to fulfil the requirements to provide QoS control in mobile IP scenarios identified by the research community and

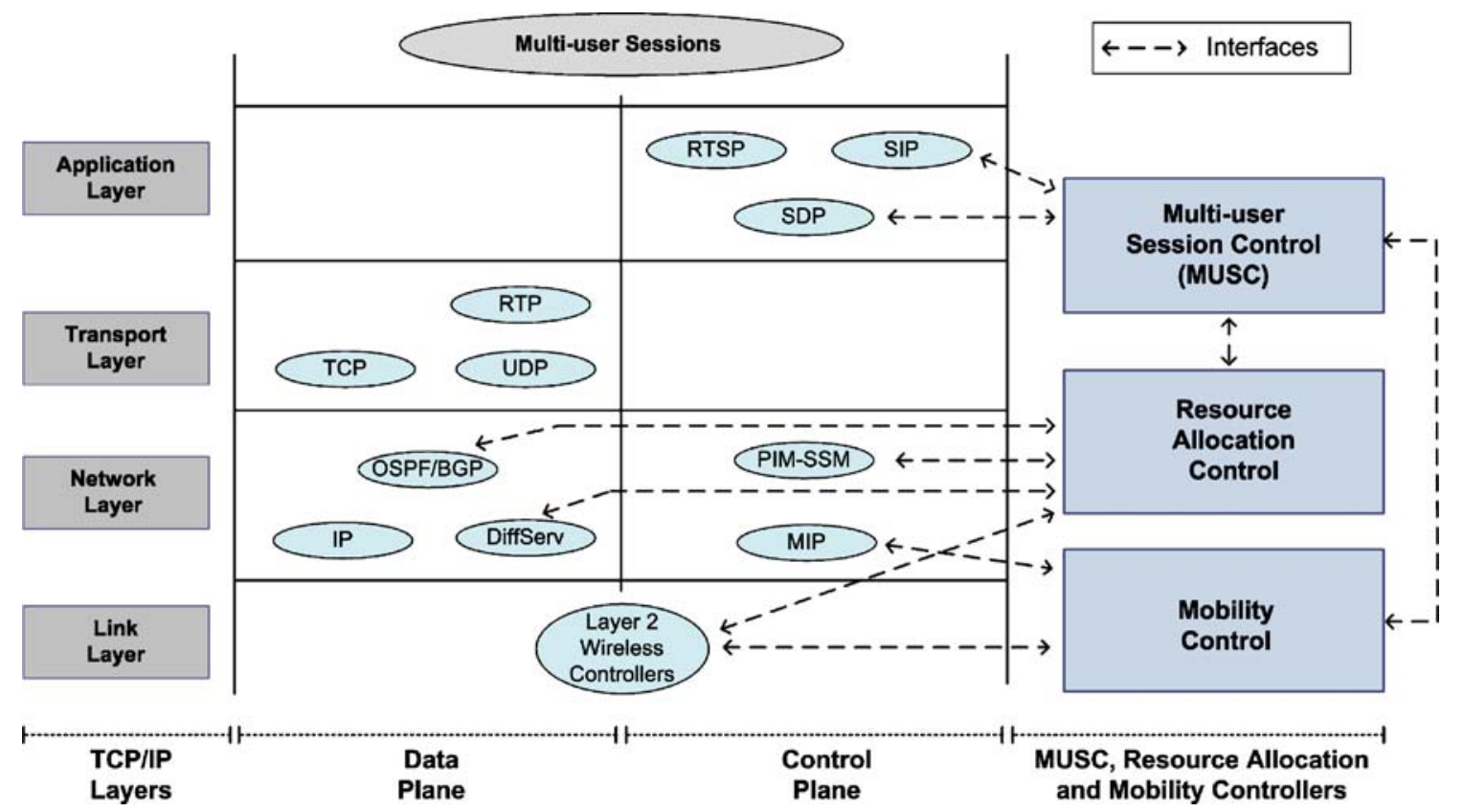

Figure 2 Relationship between the components in a TCP/IP stack view 
reported in RFC 3583 [28]. The analysis of MUSC and SEMUD to provide seamless mobility for sessions is presented in [29]. In this article, MIP is assumed as the mobility controller allowing handovers with QoS support by interacting with MUSC. MIP is a well-know IETF standard that is used by mobile operators and clients to provide the session continuity during handovers.

The Resource Allocation Control $\leftrightarrow$ DiffServ interface is used to collect information about the network services (loss, delay, jitter, and available bandwidth) by checking the PerHop-Behaviour (PHB) table on each network node from the ingress-agent (ingress point of the session in the network) to the egress-agent (exit point of the sessions in the network). During a resource reservation process, the resource controller adjusts the bandwidth reserved for the selected class in each router of the network ingress-egress path. The Resource Allocation Control $\leftrightarrow$ Layer2 Wireless Controller interface is used to retrieve information regarding the wireless classes capabilities and to interact with Medium Access Control (MAC) layer to configure the MAC classifier according to the class selected by MUSC. In order to provide QoS-aware trees, an interface with PIMSSM allows the Resource Allocation controller to activate the PIM-SSM to create and release multicast trees associated with a session. Finally, the communication between resource allocation and routing schemes, such as OSPF or BGP, assures a faster detection of re-routing events caused when a network interface goes down or comes up, allowing the interaction with MUSC to re-establish sessions on new QoS-aware paths, as reported in [30].

\subsection{Resource allocation control}

Resource allocation functions are essential to manage bandwidth resources inside and between networks, by controlling the traffic load and by accepting or rejecting incoming sessions. In the present article, Multi-Service Resource Allocation (MIRA) [31] is assumed to be the resource allocation controller. This is because MIRA coordinates the creation of QoS-aware distribution trees for multi-user sessions in environments with asymmetric routing, controls the creation of unicast flows and multicast trees associated with flows of sessions, and assures service class re-adjustment in congestion situations.

The MIRA QoS control can be performed in a centralized or decentralized mode. In the centralized mode, MIRA is implemented in a central element acting as a QoS Broker scheme to provide admission control and resource reservation for sessions. This QoS entity also maintains information about the available service classes and current bandwidth condition of each class, providing support for the MUSC QoS mapping and QoS adaptation operations. However, in order to increase the system scalability, MIRA is configured (by default) with the decentralized mode. This scheme pushes the complexity of controlling network resources to the edge MIRA agents and leaves the interior routers simple. The MIRA Protocol (MIRA-P) is used to exchange control information between MIRA agents. MIRA-P extends the NSIS QoS Signalling Layer Protocol (NSLP) [32] proposal with multicast and path-related support. This signalling protocol uses a soft-state approach to control the state maintenance for QoS-aware multicast and unicast sessions. Between networks, MIRA performs its functions based on Service Level Specification (SLS) controllers [33].

MIRA aims to build QoS-aware distribution trees associated with multi-user sessions in environments with asymmetric routing. In SSM-aware environments, MIRA provides support to a multicast routing protocol, such as PIM-SSM, to create QoS-aware multicast trees taking into consideration the QoS characteristics and current bandwidth of the path from the sender to the receiver. This goal is achieved through the update of the Multicast Routing Information Base (MRIB) with the QoS reserved-path during the resource allocation process, enabling PIM-SSM to create QoS-aware trees in environments with asymmetric routes.

In addition, MIRA uses an interface to exchange information with unicast routing mechanisms to retrieve information about the network interface towards a user or another agent. In order to contribute to the system robustness, MIRA detects re-routing events by intercepting router advertisements generated by the local routing scheme when an interface goes down or comes up (e.g., router Link State Advertisement [LSA] used by OSPF). For example, the communication between MIRA and OSPF allows the detection of re-routing events inside a network, while internetwork re-routing changes are detected through the interaction with BGP. After detecting a re-routing event, MIRA tries to accomplish a faster re-establishment of the affected sessions on the new path without damaging the current sessions. When the new path is overloaded, MUSC is requested to adapt the sessions to the current network conditions. This procedure aims to avoid session blocking and to keep sessions with an acceptable quality level.

In wireless access-networks, MIRA interacts with IEEE 802.11e and IEEE 802.16 MAC elements to control resources for service classes in wireless links. For example, in IEEE 802.11e networks, a MIRA agent is also implemented in IEEE 802.11e access points with QoS support called QoS-Access Point (QAP) [34]. In this example, DiffServ classes are assumed in the wired link from the access-agent to the QAP and IEEE 802.11e classes from the QAP to the mobile device. At the system bootstrap, MIRA (located in the access-agent) collects from the agent placed in the QAP entity the available service 
classes and resources capability of each class. During the session setup, MUSC in the access-agent, queries MIRA about the service classes in wired and wireless interfaces. After receiving the requested information, MUSC selects the wired and wireless QoS classes to be used for the session and triggers MIRA to accomplish the resource reservation process. Hence, MIRA performs the reservation in the wired interface (by configuring a DiffServ class) and signals the MIRA agent in the QAP to continue the resource reservation in the wireless link. In order to initiate a wireless QoS-aware transmission, MIRA notifies the selected wireless service to the QAP and requests the configuration of an IEEE 802.11e class (access categories) to be used for each flow of the session. The establishment of a QoS-aware transmission can be done by configuring the MAC classifier with QoS-related information described in the Traffic Specification (TSPEC), such as bit rate and packet size.

\subsection{Mapping control}

The mapping and adaptation mechanisms operate in a complementary manner. The mapping mechanism takes as input the QSPEC object of each flow and the information about the available classes (requesting MIRA). Then, it maps each flow into the suitable network classes, based on three methods: perfect, sub-perfect and hybrid matches. The mapping algorithm compares, one by one, the QoS parameters requested for each flow of the session and the list of available service classes. The Perfect Match is the preferential method, since it supports the full QoS requirements and bandwidth committed for all flows of a session. When the preferred class does not have enough available bandwidth to assure the maximum rate of the session, the QoS adaptation function is triggered, which then may decide to try a sub-perfect or a hybrid mapping. The Subperfect Match maps all flows of a session to a service class that supports QoS parameters different from the ones described in the QSPEC. This method aims to avoid session blocking and re-ordering of packets. It can be used in periods of congestion of the most suitable network class, while keeping the session full rate. The Hybrid Match assures the allocation of, at least, the high priority flows of a session to the preferred class. The remainder flows are mapped to a less significant class. This method can be used when the packet re-ordering is not crucial. For instance, it can be suitable for scheduled video and audio, where it is more important to ensure an intelligible audio flow than a perfect video.

The adaptation mechanism is triggered when the mapping process is not optimal, for instance due to a selection of an overloaded service class. Adaptation operates based on the QSPEC and on the current network conditions. The three adaptation methods are as follows: dropping or adding low priority flows of a session (rate control), requesting the use of a different QoS mapping method (remapping), or requesting extra resources to a certain service classes (readjustment) as described in Section 3.3. The process to decide which QoS mapping and QoS adaptation control strategies must be used inside or between networks can be either static or dynamic. Due to the MUSC modular approach and the use of open interfaces, the MUSC QoS control strategy can be configured by mobile providers according to their business models, inter-network agreements or on-demand via MUSC-P messages or external applications.

\subsection{Adaptation control}

The session rate control approach adapts the session by dropping or adding flows according to the priority of each flow. When the maximum bandwidth of the preferred class cannot assure the QoS committed for a low priority flow, this flow is removed from the outgoing interface and classified in the sleeping state by MUSC. Sleeping flows are awaked when the network capability becomes available again and the session full rate is supported. On the other hand, the re-mapping adaptation method requests the mapping of the session to another class (using the subperfect or hybrid mapping). The Service Class Re-adjustment method can be used to try the accommodation of the session into the preferred class, by requesting the readjustment of the maximum (extra) bandwidth assigned for the service classes. Optionally, MUSC can use a selective QoS adaptation scheme to minimize the number of blocked sessions, while keeping current sessions with an acceptable quality. This adaptation algorithm selects an already admitted session (or a set of sessions) and then decreases their quality (until the minimum acceptable rate specified in the SOBJ) by dropping low priority flows or requesting the re-mapping of those flows into other classes. This selection can be done randomly or using other fairness-based "adaptation weight" scheme, such as:

- High-rate: sessions with high-rate are selected

- Popularity-based: sessions with small audience (number of users) are selected [35]

- Price-based: sessions with low price or with monetary incentives in case of degradation are chosen [36]

If more than one session has the same adaptation weight, a random selection is applied based on the selective adaptation limit $\left(\lambda_{\text {adp }}\right)$. The selective adaptation limit represents the percentage of admitted sessions that can be degraded. It is clear that with an aggressive limit $\left(\lambda_{\text {adp }}=\right.$ $100 \%$ ), more "new" sessions can be accepted. The fairnessbased algorithm as well as the selective adaptation limit can 
be configured manually by the operator (according to its business model or internetworking agreements) or ondemand based on historical usage data and traffic pattern. When an admitted session terminates or moves, the corresponding resources are released and the quality level of adapted sessions is enhanced again.

\subsection{Signalling associated with handovers}

MUSC-P is used to exchange information between MUSC agents using a soft-state approach to maintain per-session and per-flow state (including the QSPEC of each flow, which is necessary in re-routing or mobile events). MUSC$\mathrm{P}$ is being specified based on the NSIS framework, in which it can be included as an extra NSLP. MUSC-P operates in a receiver-driven approach, since it is triggered at the access-agent (agent located in wired or wireless access-router). MUSC-P is source-initiated, since it used to start the QoS configuration of MUSC agents at the agent nearest to the source, or at the first agent in the path towards the source that contains the requested session. When MUSC is triggered by MIP-alike approaches, only source-initiated functions are done to control the quality level of sessions from the home agent (source in this case) to the moving receivers. MUSC-P aims to reduce signalling due to handovers as suggested in the NSIS working group [37].

MUSC-P supports three message types to exchange control information among MUSC agents, namely, the SessRequest, SessResponse and SessRefresh. The SessRequest message is sent, with the IP router alert option, by a MUSC access-agent towards the agent where the requested session is activated. The destination of the SessRequest message is the agent closest to the source of the signalled session. However, this message can be stopped by the first agent discovered along the path towards the source which has the requested session (i.e., the agent that has another branch point for the session). The SessResponse message is used to install the session from the agent in which the session is found towards the MUSC access-agent (or HA in case of MIP) where the IP address of each downstream agent is provided by MIRA. In addition, SessRefresh messages are sent periodically by downstream MUSC agents to upstream agents that are the ingress point of the sessions to be refreshed. If the state of a session is not refreshed within a certain period of time by using SessRefresh messages, its state is removed by soft-state.

Figure 3 shows an example of the message sequence used by MIPv4, MUSC-P and MIRA-P to provide the continuity of an ongoing session with QoS support. It is assumed that the mobility is controlled by a MIP bidirectional tunnelling approach, where MUSC agents are collocated with MIRA at the edges of the networks.
Furthermore, HA and Foreign Agent (FA) are also placed in the unique ingress point of access-networks. Since handover controllers are not the focus of this article, the use of MIPv4 is assumed in Fig. 3. If MIPv6 would be supported, the FA would not be required on the data path. In order to reserve QoS resources in a service class selected by MUSC, a MIRA-P Reserve message is sent to notify MIRA agents (core and edge routers) in the downstream path regarding the amount of resources required for each flow. Additionally, an upstream MIRA-P Response message is triggered in the egress-agent or access-agent to notify the ingress-agent about the result of the requested operation (admission control and resource reservationaccepted or not).

The handover process starts when the mobile node sends a MIP Agent Solicitation message and receives a MIP Agent Advertisement message to determine whether it is on its home network or on a foreign network. These messages are not used if HA and FA are configured to advertise their presence via agent advertisement messages. Upon moving to a foreign network and obtaining a care-of-address of the foreign agent, the mobile node registers its new address with its home agent through the exchange of MIP Registration Request and Registration Reply messages (possibly via the foreign agent). After the registration process, the MIP HA notifies MUSC to control the session quality level on the new path (which includes the QoS-aware tunnel between the HA and FA). Based on the session identifier associated with the moving node and supplied by the HA, MUSC retrieves the correspondent QSPEC object stored in the ingress agent. Subsequently, the interaction between MUSC and MIRA allows the creation of a QoS-aware path to be used by the session along the path from the HA to the mobile node. All packets addressed to the mobile node's home address are intercepted by its HA and tunnelled to its foreign network (by using the node's care-of-address). In the foreign network, the FA receives the tunnelled packets and sends them to the mobile node with QoS support.

\section{Example of overall functionality}

This section shows examples of MUSC functionality to accomplish QoS mapping and adaptation for multi-user sessions due to an inter-network handover. The mobility is controlled by a MIP-based bi-directional tunnelling approach, in which MUSC agents are collocated with MIRA at the edges of the networks. Additionally, HA and FA are also placed in the unique ingress point of access-networks. The scenario of Fig. 4 describes three networks with different QoS models, where one multi-user session (S1) 


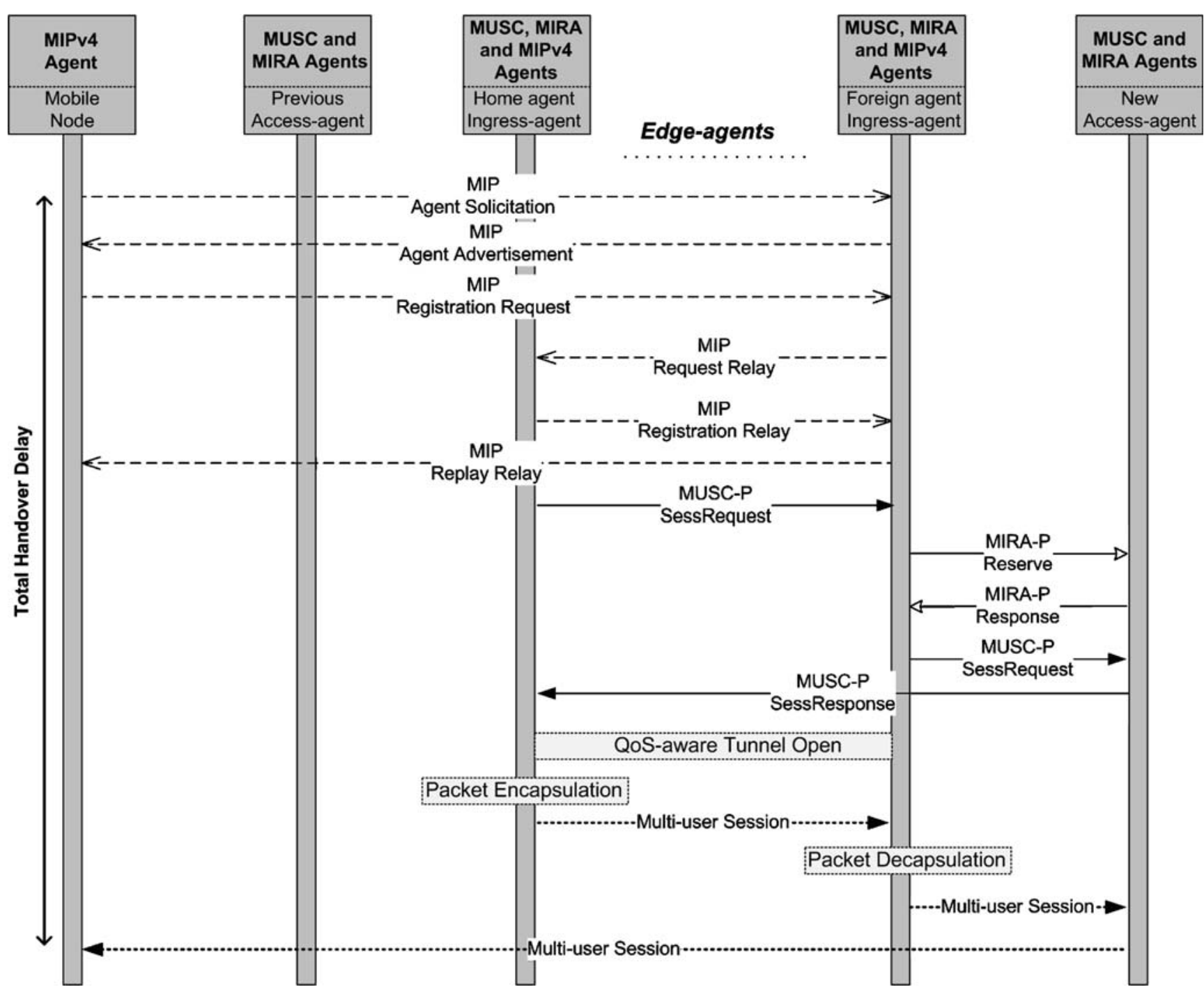

Figure 3 Message sequence used by MIPv4, MUSC-P and MIRA-P in an inter-network handover

with three flows is multicasted toward two mobile receivers (R1 and R2). Thus, when R2 moves to the access-agent N.3.1, it receives a router advertisement message and acquires a care-of-address on the foreign network. After that, R2 registers its new address with its HA through the exchange of registration messages. Upon finishing the registration process, the $\mathrm{HA}$ placed in $\mathrm{N} 1$ notifies the MUSC agent N.1.3 to control the session quality level on the path towards N.3.1 (which includes QoS-aware tunnels between the HA and FA). Based on the session identifier associated with R2 and supplied by the HA, MUSC in agent N.1.3 consults its state and retrieves the correspondent QSPEC object. After that, MUSC triggers MIRA to query information about the available classes and their QoS characteristics of the inter-network link between the agents N.1.3 and N.2.2.

Based on the response and QoS parameters collected in the QSPEC object, the MUSC mapping mechanism compares, one by one, the QSPEC object parameters of each flow with the capability of each class. After a successful match, it selects the most suitable service class for each flow. According to the priority of each flow, MIRA is triggered to configure the required bandwidth in the preferred class (inter-network path from N.1.3 to N.2.2). After the resource reservation process, including admission control functions, MUSC is triggered and controls the quality level of the session on the remainder path. Hence, a MUSC-P message is sent to the agent N.2.2, which verifies the QSPEC object of each flow and interacts with MIRA in the same way as illustrated before.

According to the MIRA response and QSPEC object, MUSC selects the appropriate network service and triggers MIRA to configure the required bandwidth in the selected class (from N.2.2 to N.3.2). After admission control procedures, MUSC is notified because the preferred service class has not enough resources to accommodate the less 
Figure 4 Example of MUSC re-mapping adaptation control

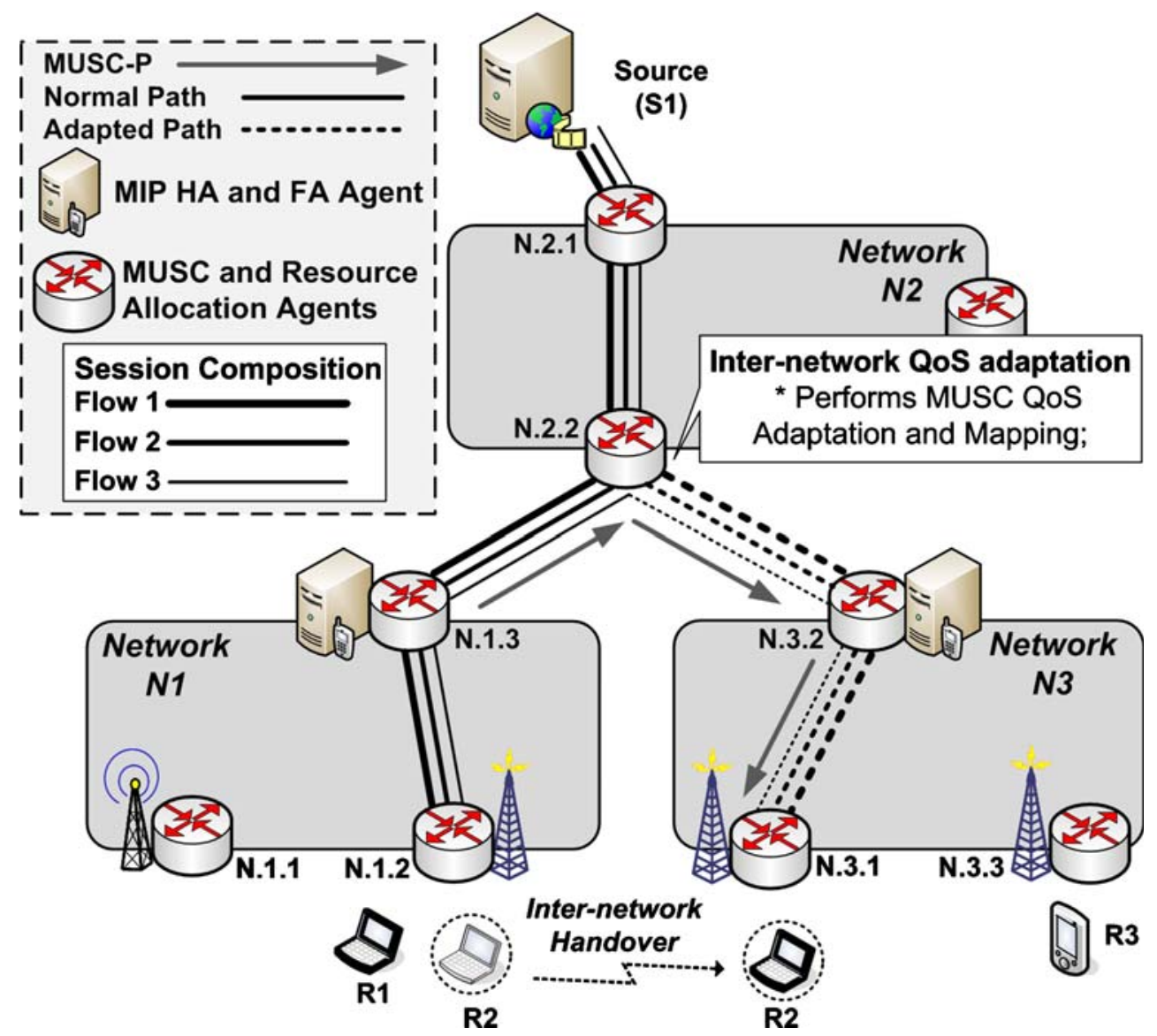

priority flow of the session. Since MUSC supports flexible methods to control the session quality level, it can be configured by operators with different profiles. For example, based on the local configuration, MUSC uses a combination of the Re-mapping adaptation and Hybrid Match mapping methods to decrease session blocking probability. Thus, MUSC requests the allocation of the highest priority flows into the preferred network class while the remainder flows are mapped to a less important class. After all the QoS control operations performed by MUSC and MIRA in the agent N.2.2, MUSC-P is triggered to signal the remainder downstream agents. All agents along the downstream adapted path will accomplish the same QoS control operations, as described before, in order to resume the creation of a QoS-aware path for the ongoing session. In agent N.3.1, MUSC selects the preferred class for the session based on the wireless classes (e.g., IEEE 802.11e or IEEE 802.16) and resources.

Alternatively, Fig. 5 depicts a dynamic session adaptation control started at agent N.2.2, where MUSC is configured to adapt the session quality level by dropping low priority flows. Thus, upon selecting the preferred service class between N2 and N3, MUSC is triggered by MIRA to adapt the session. Since the adaptation mechanism is configured to adjust the number of flows, Flow 3 is removed from $\mathrm{S} 1$ and the remainder downstream agents are signalled to control quality level only for Flow 1 and Flow 2. MUSC agent N.2.2 keeps Flow 3 state as sleeping, using this state to increase the number of flows of $\mathrm{S} 1$ when network resources in the preferred class become available again. For instance, when the bottlenecked inter-network class is freed, MUSC agent N.2.2 puts the Flow 3 in "awake state," triggers the resource controller to reserve resources for this flow and signals downstream agents to provide QoS control for Flow 3 towards R2.

In order to avoid the waste of network resources associated with Flow 3, a MUSC-P message is sent from the agent N.2.2 to the agent N.1.3. This message triggers MUSC to release the resources used by Flow 3. Upon removing Flow 3 state, MUSC notifies MIRA and the HA to delete the state associated with Flow 3 on the new path. The resources allocated for this flow in the end-to-end old path are not removed, because the agent N.1.3 has another receiver for S1 using Flow 3.

\section{Performance evaluation}

Performance evaluation of the MUSC proposal was carried out based on simulation and prototype experiments. The 
Figure 5 Example of MUSC drop/add adaptation control

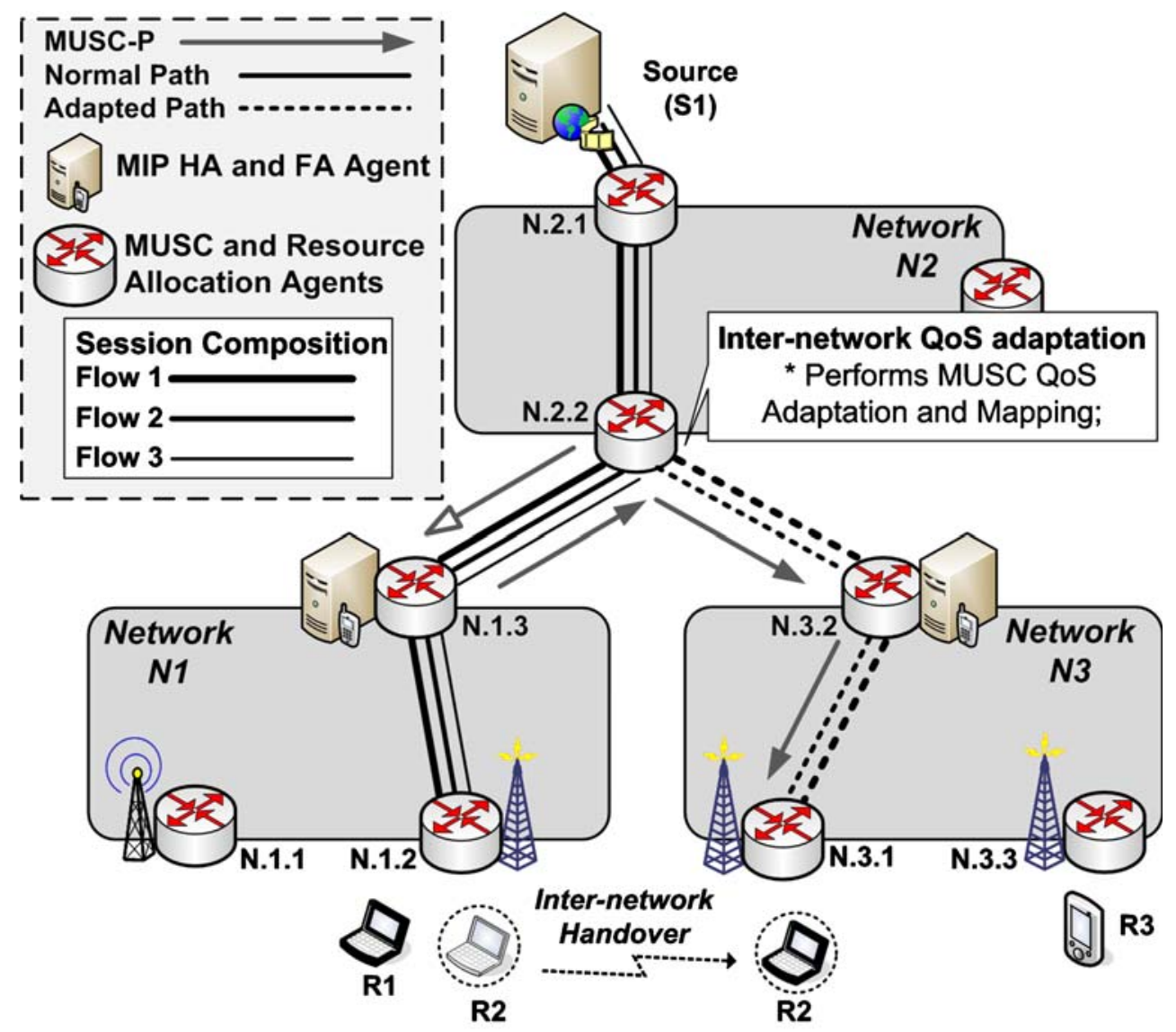

objectives of the simulation are the following: (1) analyze the MUSC latency to control the session quality level on new paths; (2) analyze the impact on receivers' expectation by measuring throughput and one-way delay of scalable multi-user sessions as well as the perceived quality of a video sequence by verifying the PSNR, MOS and SSIM of a non-scalable multi-user session with and without QoS adaptation; (3) analyze the session blocking probability with different MUSC selective dropping limits., Additionally, in order to confirm the results achieved during the simulation and to verify the MUSC applicability in real networks, a prototype is used to analyse the throughput and RTT delay observed by receivers, in home and foreign networks, with and without QoS adaptation.

The following four main QoS adaptation profiles used to evaluate the MUSC proposal are (based on a static configuration): The N_ADP profile, in which no QoS adaptation method is used. In this case, the session is blocked if the complete set of QoS requirements cannot be assured in the preferred class; The ADP_Drop profile, in which session quality is controlled by dropping and adding flows; The ADP_Hyb profile, in which flows with high priority are mapped to the preferred class and flows with lower priority are re-allocated to a less important class. In this profile, the session is blocked only if the full rate of high priority flows cannot be assured in the preferred service class; and The ADP_Sub profile, in which all flows of a session are re-mapped to a less important class. In this case, the session is blocked if the full rate of the session cannot be assured in the misplaced class.

As suggested in [38], the maximum reservation threshold of each class is $20 \%$ for Premium, $20 \%$ for Gold, $20 \%$ for Silver and the remaining $40 \%$ for Best-effort. The Premium class is configured with the following QoS parameters: loss, delay and jitter tolerance (e.g., as suggested for DiffServ Expedited Forwarding (EF) and IEEE 802.11e AC Voice alike classes).

In order to verify the impact of this proposal with different kinds of sessions, the evaluation is performed using scalable and non-scalable multi-user sessions. The former is composed of three flows with the same QoS requirements. Although MUSC can handle any number of flows, three flows allow a good trade-off between quality and bandwidth, and additional flows only provide marginal improvements [39]. Additionally, each flow has different priorities and exponential rates, which are common in scalable CODECs [39]. Each one of the three flows has a Constant Bit Rate (CBR) of 32, 64 and $128 \mathrm{~kb} / \mathrm{s}$, starting from the most important to the less important one, respectively. On the other hand, a Variable Bit Rate (VBR) non-scalable multi-user session with an average rate of $67 \mathrm{~kb} / \mathrm{s}$ rate is used. The non-scalable session consists of 
a 960 frames video sequence denominated "Mother and Daughter [40]" with YUV format, sampling 4:2:0, dimension $352 \times 288$, that were compressed through a MPEG4 codec, and sent with a $30 \mathrm{frame} / \mathrm{s}$ rate. The Group of Pictures (GOP) of the sequence is composed by 30 frames and each frame is fragmented in blocs with 1,024 bytes. This evaluation resorts in the Evalvid tool [41].

It is assumed that intolerance to loss is the major requirement of the session and that a loss limit of $2.5 \%$ is the maximum degradation allowed in the QSPEC. This limit is based on previous studies [42], where it is presented that in MPEG-2 with Signal-to-Noise Ratio scalability, 5\% of losses in the most important flow introduces $100 \%$ of losses in all other flows. Moreover, real-time sessions require a one-way delay lesser than $100 \mathrm{~ms}$. The QSPEC object of each flow of a session is generated following a Poisson distribution.

\subsection{Simulation}

The simulation was done using the Network Simulator 2 (NS2) and the topology was generated randomly by BRITE. The first set of experiments aims to analyze the impact of MUSC due to inter-network handovers, while the second set of experiments aims to verify MUSC behaviour due to intra-network mobility. Each edge MUSC is put together with MIRA, being the latter responsible to provide notification about available classes, admission control and service class configuration. In the ingress points, MUSC is also collocated with MIP HA and FA, where MIP HA manages the mobility and triggers MUSC to control the session quality on new paths. The mobility is controlled by MIPv4 and receivers are connected to IEEE 802.11e wireless access-agents.

\subsubsection{MUSC behaviour due to inter-network handover}

The simulation scenario used in this set of experiments is composed by three networks: one of them hosts the sources while the other two host the receivers as presented in Fig. 6. Each network has 16 interior routers and three edges, where DiffServ and IEEE 802.11e are configured as the QoS models. The propagation delay is assigned by BRITE according to the distance of each device. The first experiment aims to analyze the behaviour of MUSC due to inter-network handovers, where the bandwidth capacity of intra and inter-network links is 100 and $10 \mathrm{Mb} / \mathrm{s}$, respectively, while wireless links have a bandwidth of $11 \mathrm{Mb} / \mathrm{s}$. From the handover point of view, the home (Network N1 in Fig. 6) and foreign (Network N3 in Fig. 6) networks have 10 and 20 receivers, respectively, where each one subscribed one scalable multi-user session following the Poisson distribution (five and ten receivers in each access-router in the home and foreign networks,
Figure 6 Topology used in the first simulation experiment

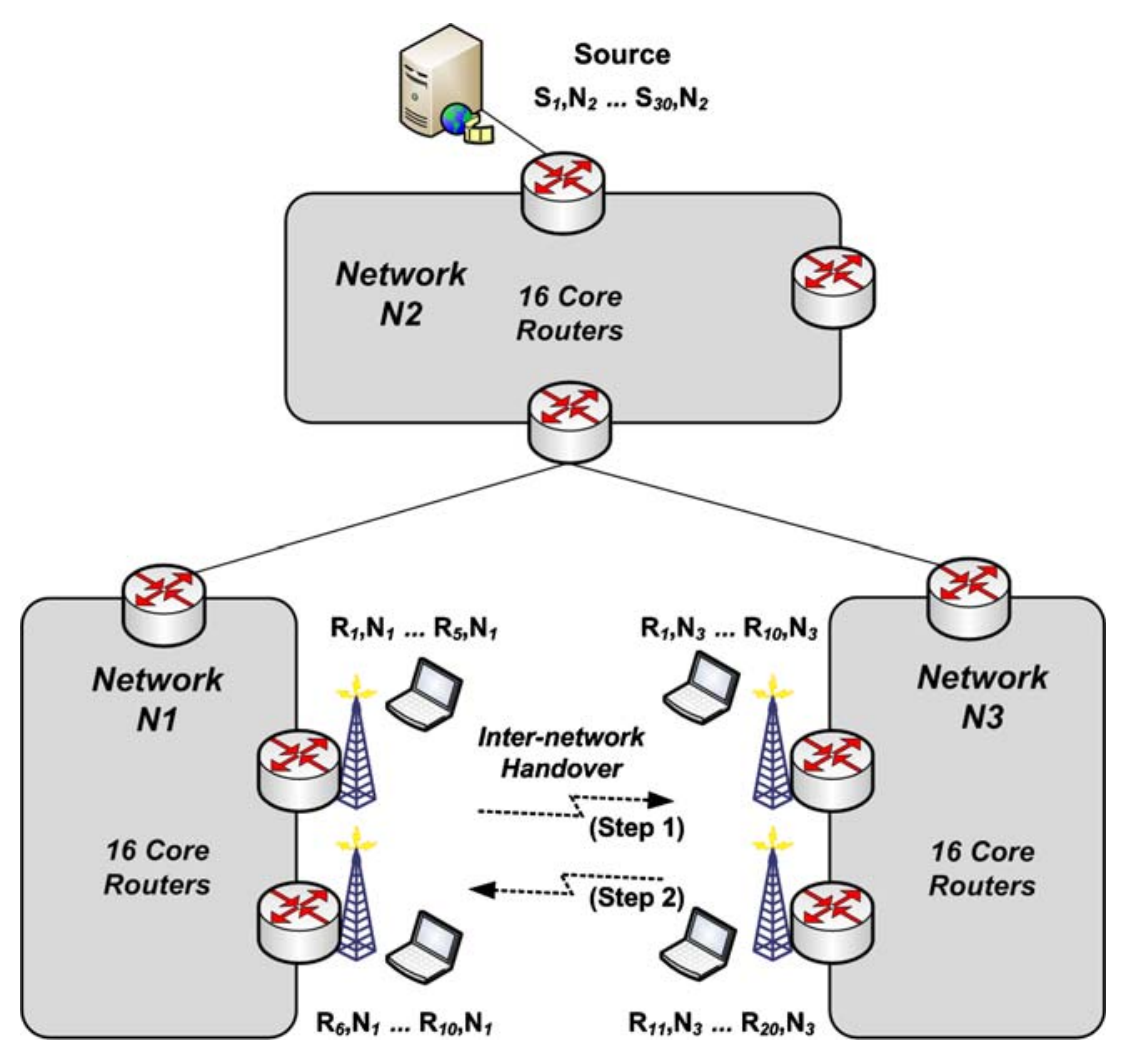


respectively). To simplify the experiment, each receiver moves to an access-agent in the foreign network $30 \mathrm{~s}$ after its subscription (step 1) and returns to its previous accessagent in the home network $70 \mathrm{~s}$ latter (step 2). The movement pattern follows a constant speed of $30 \mathrm{~m} / \mathrm{s}$. Since inter-network adaptation is the focus of this experiment, the bandwidth required for all the sessions exceeds in $12 \%$ the amount of resources allocated for all classes in the inter-network link from the network N2 to the network N3. Note that congestion higher than $12 \%$ in the inter-network link causes blocking of $100 \%$ of incoming sessions.

The results reveal that during handovers MUSC introduces an average latency of $15.8 \mathrm{~ms}$ to configure its QoS mapping and adaptation mechanisms along new session paths. This value is an average of all handovers (ten moving receivers) and represents $0.1 \%$ of the delay consumed during the mobility process, which can be considered negligible. This way the use of MUSC contributes for the creation of QoS-aware sessions and does not introduce high latency in handover times.

Figure 7 depicts the average throughput measured by the moving receivers at the foreign cluster when the optional selective adaptation is disabled $\left(\lambda_{\mathrm{adp}}=0 \%\right)$. In all situations, the session associated with R10 is refused due to unavailability of resources in the network. The N_ADP profile keeps the session full rate only for $40 \%$ of the receivers, whilst $60 \%$ of the sessions are blocked. Using the profile ADP_Drop, MUSC controls the session quality level and keeps them within acceptable quality, because only the least important flow of the session is affected in handovers. Thus, $90 \%$ of the receivers access the sessions in the foreign cluster. This is, R2, R3, R4 and R7, get the session full rate, while R1, R5, R6, R8 and R9 receive only Flow 1 and Flow 2. When ADP_Hyb and ADP Sub are used, the session full rate is assured through the use of resources available in other service classes. The ADP_Hyb profile keeps the session full rate for $60 \%$ of receivers, in which three sessions are re-mapped to other classes. The ADP_Sub adapts $20 \%$ of sessions to less important classes, but it assures the session full rate for R1, R2, R3 and R4. The drawback of these last two profiles is the fact that adapted sessions consume network resources of other classes, which increases the call blocking of sessions best suited to those classes. For instance, when ADP_Hyb and ADP_Sub are configured, the session associated with R7 is refused because other misplaced sessions are using resources allocated for its preferred class.

Figure 8 shows in detail the throughput measured in R1 and R6 with all the profiles. The session associated with R6 is blocked in the foreign network when the ADP_Sub and N_ADP profiles are configured, while the session of $\mathrm{R} 1$ is refused in the foreign network when the N_ADP profile is being used. For a better explanation, Table 1 summarizes the throughput measured in R6 in home and foreign networks.

In the home network, the session full rate is assured for R1 and R6, since there are available resources to accommodate the flows in the preferred service class. The throughput is zero when R1 and R6 are moving to the foreign network (R1: 32.75 to $52.5 \mathrm{~s}$ and R6: 33.2 to $53 \mathrm{~s}$ ) and during their return to the home network (R1: 72.75 to $86.5 \mathrm{~ms}$ and R6: 73.2 to $86.8 \mathrm{~s}$ ). The ADP_Drop adapts multi-user ongoing sessions according to the priority of each flow, which reduces the impact on the session quality level at the foreign network and
Figure 7 Average throughput in moving receivers at the foreign network

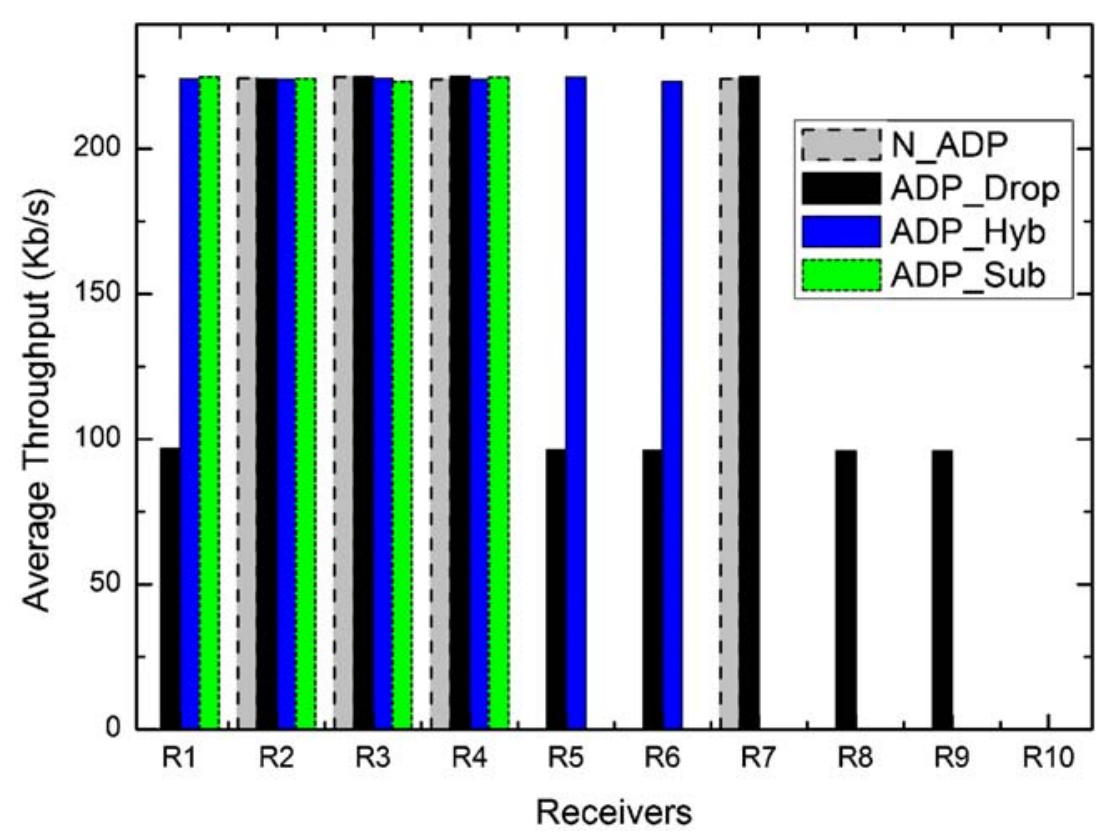


Figure 8 Throughput and latency in R1 and R6 when all profiles are being used

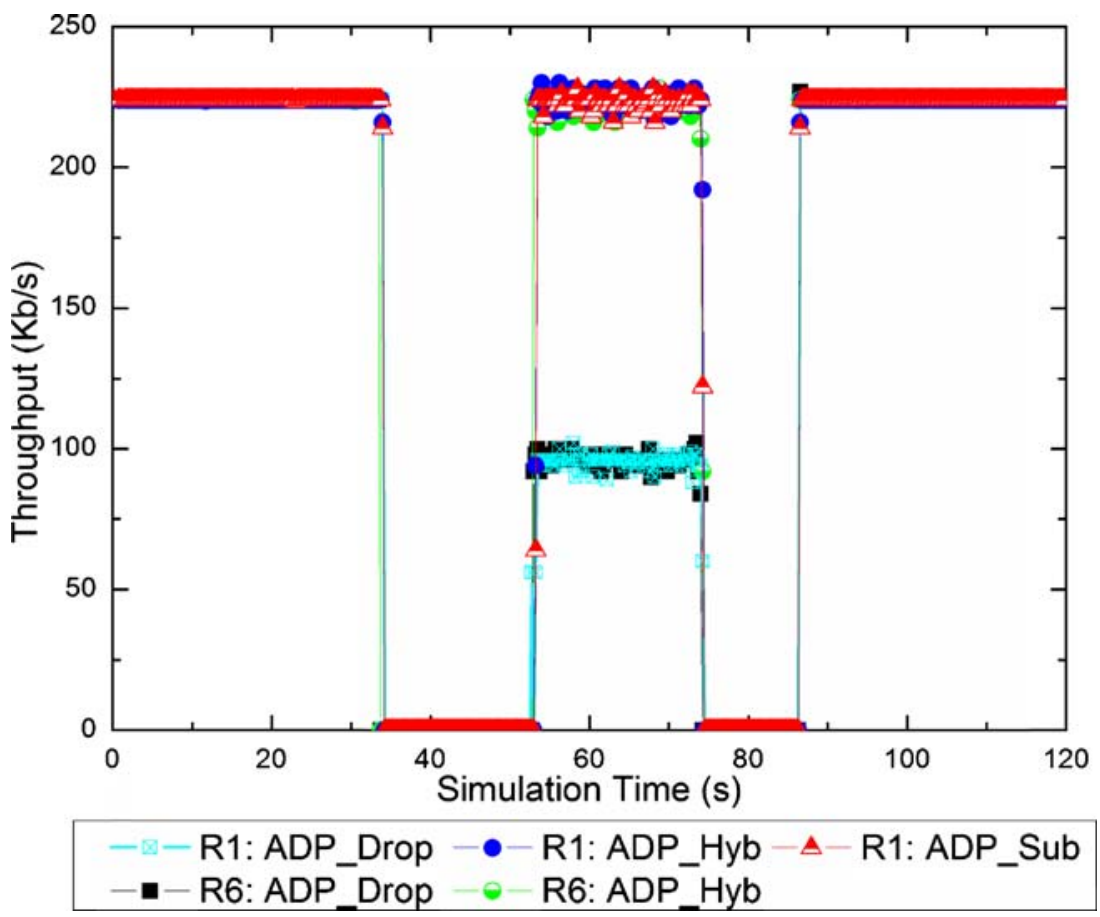

keeps the full rate of Flow 1 and Flow 2. The Flow 3 of the sessions associated with R1 and R6 is put in sleeping state and it is awaked only after the return of the mobile devices to their home network. In comparison with the ADP_Drop, the ADP_Hyb improves the session quality level, because the full rate of each flow is assured by using the available resources of another service class to accommodate the Flow 3.

As explained before, the session full rate is assured by the ADP_Hyb and ADP_Sub profiles, while the one-way delay is degraded. The latter is an important QoS metric especially for real-time sessions and must be kept with an acceptable quality level. The average one-way delay in moving receivers, at the foreign network, is presented in Fig. 9 whilst Table 2 summarizes the one-way delay in R1 with all profiles. The one-way delay of R2, R3 and R4 are the same with all profile because their sessions were mapped into the preferred classes (not adapted).

On average, the one-way delay in the home network, where the session is mapped into the preferred class, is $29.7 \mathrm{~ms}$. In the foreign network, the one-way delay is increased due to the use of MIP tunnels to encapsulate/ decapsulate the packets of the session and the session mapping in less suitable classes. The session adaptation to a service class that offers a different delay tolerance also influences the one-way delay. Thus, R1 needs to wait on average 70.02, 72.98 and $77.07 \mathrm{~ms}$ when the ADP_Drop, ADP_Hyb and ADP_Sub profiles are being used, respectively. In the worst case, as occurs with the ADP Sub, R1 waits approximately $5 \%$ and $10 \%$ more to get the session, when compared with the ADP_Hyb and ADP_Drop methods, respectively. However, this value remains accept- able for the ongoing session as required in the QSPEC object. If the maximum one-way delay in misplaced classes is not assured, the session is blocked.

In order to increase the number of admitted ongoing sessions in the foreign access-network, a second set of experiments was performed to evaluate MUSC with a selective dropping scheme of $25 \%\left(\lambda_{\mathrm{adp}}=25 \%\right)$ using the same previous inter-network mobility scenario. However, the number of moving receivers in each of the two accessagents in the home access-network is incremented to ten (total of 20 moving receivers).

The results reveal that MUSC reduces the quality level of $25 \%$ of the admitted sessions in a class during the congestion period (during the attachment of receivers in the foreign access-network), while the full rate of the "degraded sessions" is assured when the resources become available again (from the home access-network). The less

Table 1 Throughput in R6 measured at the foreign network for all profiles

\begin{tabular}{llc}
\hline Throughput & ADP_Drop $(\mathrm{kb} / \mathrm{s})$ & ADP_Hyb $(\mathrm{kb} / \mathrm{s})$ \\
\hline Flow 1-Max & 36.02 & 34.40 \\
Flow 1-Min & 28.02 & 22.00 \\
Flow 1-Ave & 31.47 & 31.03 \\
Flow 2-Max & 66.12 & 66.00 \\
Flow 2-Min & 60.04 & 58.02 \\
Flow 2-Ave & 64.15 & 62.43 \\
Flow 3-Max & - & 132.08 \\
Flow 3-Min & - & 120.00 \\
Flow 3-Ave & - & 126.66 \\
\hline
\end{tabular}


Figure 9 Average one-way delay in moving receivers in the foreign network

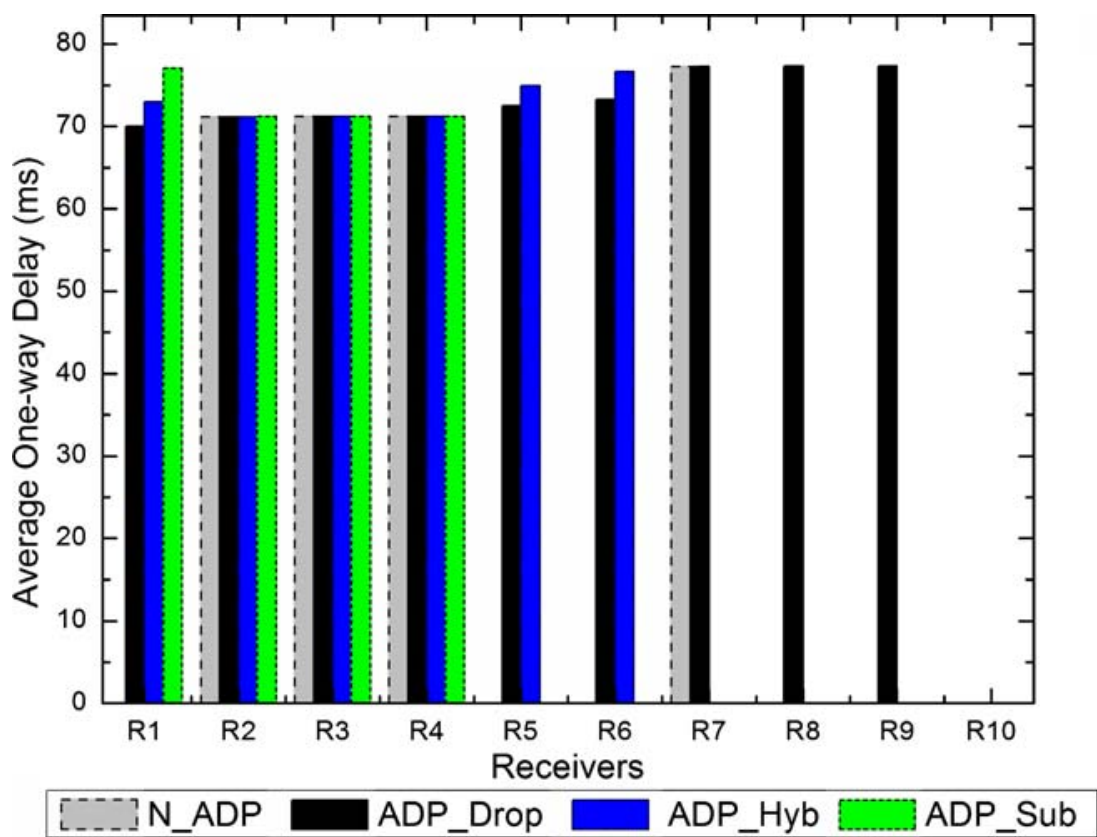

important flow of current sessions is put in sleeping state and the rate of each degraded session is of approximately $96 \mathrm{~kb} / \mathrm{s}$. However, 85\% of ongoing sessions were accepted being $24 \%$ with full rate $(\sim 224 \mathrm{~kb} / \mathrm{s}), 64 \%$ with the two most important flows $(\sim 96 \mathrm{~kb} / \mathrm{s})$ and $12 \%$ of the re-routed sessions were accommodated only with the highest priority flow $(\sim 32 \mathrm{~kb} / \mathrm{s})$. The number of accepted sessions increases $35 \%$ in comparison to the same scenario without selective adaptation (or $\lambda_{\text {adp }}=0 \%$ ). Naturally, since $25 \%$ of the admitted sessions are degraded, the per-session average throughput is lower, being the cost to pay for the admission of an additional number of sessions (session average throughput of all the sessions in the foreign accessnetwork). The average per-session throughput is 198.48 and $161.12 \mathrm{~kb} / \mathrm{s}$ when the $\lambda_{\text {adp }}$ is $0 \%$ and $25 \%$, respectively. Since the session quality degradation is done according to the flow importance, the impact on its quality level is minimized.

The analysis of the previous scenario with a MUSC aggressive selective dropping of $50 \%\left(\lambda_{\text {adp }}=50 \%\right)$ was also performed. The results show that all ongoing multi-user sessions are accepted, while degrading the quality level of already admitted sessions. Despite the use of a $\lambda_{\text {adp }}=50 \%$, only $43 \%$ of admitted multi-user sessions were degraded to accepted $100 \%$ of moving sessions. This result is due to the fact that releasing resources of $43 \%$ of the current sessions was enough to support $20 \%$ of ongoing sessions with full rate, and $80 \%$ of sessions with the two most important flows. The average per-session throughput in the foreign access-cluster is $148.86 \mathrm{~kb} / \mathrm{s}$, which represents a reduction of approximately $8 \%$ compared to the experiment using a $\lambda_{\mathrm{adp}}=25 \%$.

\subsubsection{MUSC behaviour due to intra-network handover}

In order to verify the impact of MUSC on the quality perceived of a real multimedia video due to an intranetwork handover, the MOS, PSNR and SSIM metrics of the video were analysed. The MOS evaluation method proposed by ITU [43], ANSI [44] and MPEG [45] is used to quantify the video quality based on the human quality impression (subjective method) and it is given on a scale from 5 (best) to 1 (worst). The SSIM parameter is an objective method which compares information about luminance, contrast and structural similarity between original and processed frames and combines them into a result value ( 0 represents the maximum distortion, while 1 indicates the minimal distortion) [46]. Since the human visual system is sensitive to structural distortion inside a frame, quality assessment with SSIM parameter is an important subjective method to analyze the impact in the video quality assessment [47]. The PSNR is a well-known objective method to evaluate the quality of the received sequence and to map the video to the MOS scale as presented in Table 3. Considering frames with $M \times N$ pixels

Table 2 One-way delay of R1 in the foreign access-network for all profiles

\begin{tabular}{lccc}
\hline $\begin{array}{l}\text { One-way } \\
\text { delay }\end{array}$ & ADP_Drop (ms) & ADP_Hyb (ms) & ADP_Sub (ms) \\
\hline Max & 80.76 & 83.82 & 86.59 \\
Min & 65.46 & 67.94 & 71.00 \\
Avg & 70.02 & 72.98 & 77.07 \\
\hline
\end{tabular}


Table 3 PSNR to MOS conversion

\begin{tabular}{ll}
\hline PSNR $(\mathrm{db})$ & MOS \\
\hline$>37$ & 5 (Excellent) \\
$31-37$ & 4 (Good) \\
$25-31$ & 3 (Fair) \\
$20-25$ & 2 (Poor) \\
$<20$ & 1 (Bad)
\end{tabular}

and 8 bits/sample this metric is defined through the expression 1:

PSNR $=20 \log _{10}\left(\frac{255}{\sqrt{\frac{1}{M \times N} \sum_{1=0}^{M-1} \sum_{j=0}^{N-1}\|\mathrm{Ys}(i, j)-\mathrm{Yd}(i, j)\|^{2}}}\right)$

In this expression, $\operatorname{Ys}(i, j)$ designates the pixel in the position $(i, j)$ of the original frame and the $\mathrm{Yd}(i, j)$ refers to the pixel located in the position $(i, j)$ of the reconstructed frame.

In this experiment, a mobile receiver moves to a new access-agent in the same access-network, where the quality level of the non-scalable session is controlled by MUSC. Two situations are considered in the foreign access-agent: The first (a) assumes availability of wireless resources to accommodate the session into the preferred class, while the second (b) assumes unavailability of wireless resources to assure the session full rate into the preferred class (conges- tion of approximately $15 \%$ is assumed in the preferred class caused by concurrent traffic). In addition, a new evaluation profile is included, called $n$ adp_loss profile. The main difference to a N_ADP profile is the fact that the session is accepted even when its full rate is not assured and intolerant frame losses are expected in the selected class (admission control and QoS adaptation are disabled). Based on the two referred situations, MUSC is evaluated with three profiles: N_ADP (used as benchmark profile_-case a), n_adp_loss (case b) and ADP_Sub (case b) profiles. The last aims to keep the session quality perceived at an acceptable level based on the sub-perfect method.

Figure 10 presents the results obtained concerning the PSNR of each video frame when MUSC is configured with N_ADP, ADP_Sub and n_adp_loss profiles. The results reveal that before the handover, the average PSNR of the video sequence is of $41 \mathrm{db}$, which represents the value 5 in the MOS scale. After the handover, the average PSNR with N_ADP profile is of $39.81 \mathrm{db}$, where the video remains excellent according to the MOS scale. Several frames $(\sim 380$ to 387 ) are lost during the first second after the handover, which damages the decoder/recover of other frames due to the frame's inter-dependency (I, P and B frames) and reduces the PSNR. When the $n$ _adp_loss profile is configured, the session quality level is degraded from excellent in the home agent to bad in the foreign agent according to the MOS scale, where the average PSNR in the foreign agent is of $19.6 \mathrm{db}$. The benefits of MUSC QoS adaptation (ADP_Sub) in the video sequence is observable in Fig. 10, where it keeps the ongoing session with excellent quality level. The average PSNR in the foreign agent is of $38.8 \mathrm{db}$. The PSNR of the frames 380 to 386,690 to 700,812 to 815 and 902 to 906 is
Figure 10 Delay and latency in R1 with all profiles

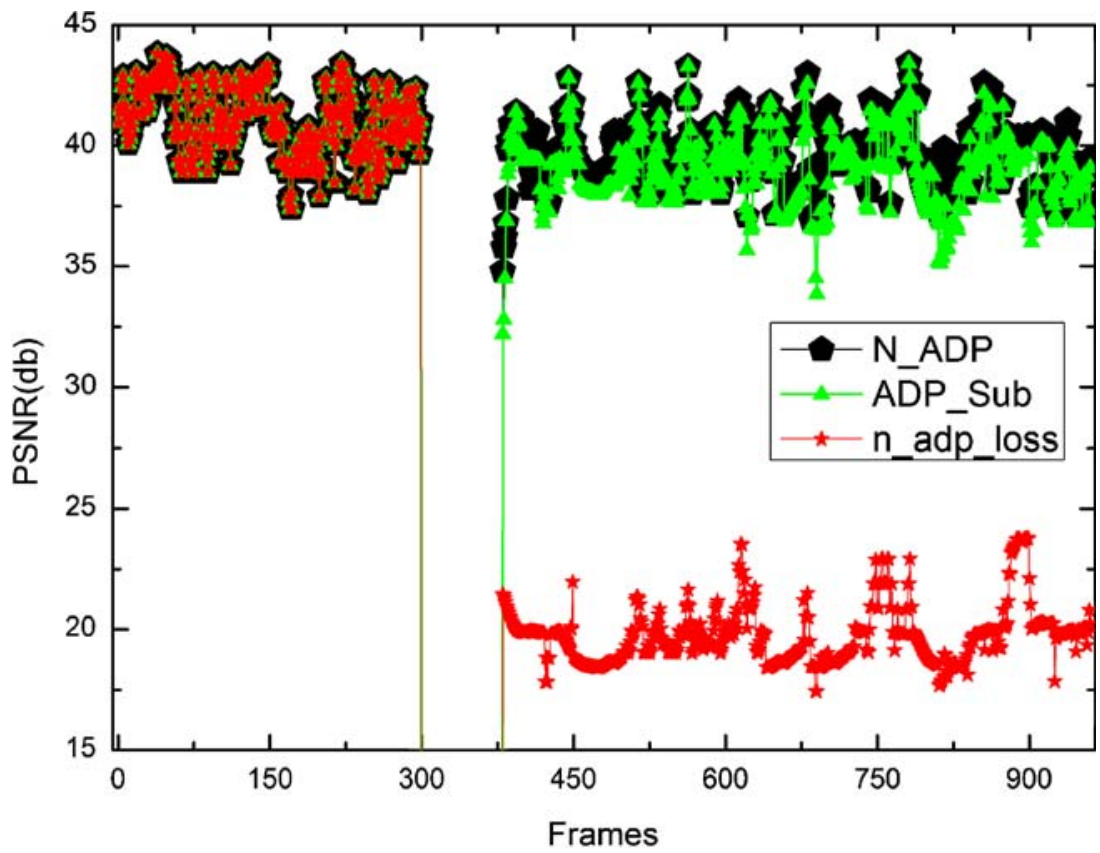


Table 4 Some frames of "Mother and Daughter" in the foreign agent based on n_adp_loss and ADP_Sub profiles

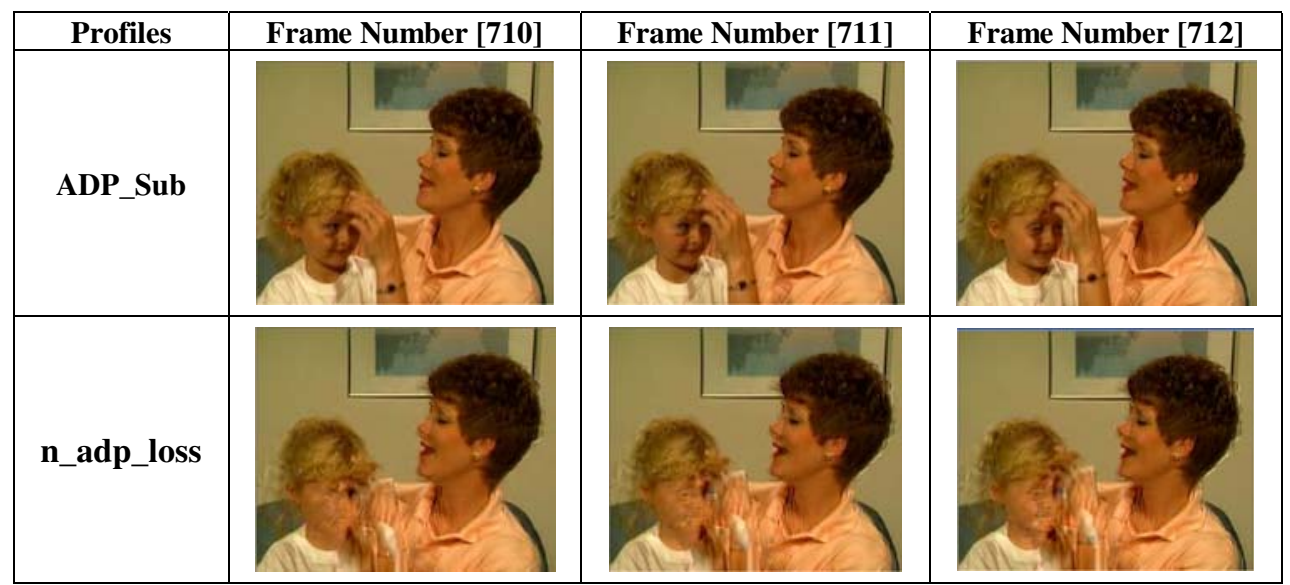

reduced due to the lost of frames in the middle of the video sequence and the MOS is considered good.

When the QoS adaptation mechanism is used, the PSNR in the foreign agent is reduced in approximately $2.5 \%$ compared with the N_ADP using a perfect match mapping, which represents very low impact on the user's experience. The video degradation and poor quality is visible when the system is configured with the $\mathrm{n}$ ada loss profile as illustrated in some frames of Table 4. When $n$ adp loss method is analyzed, the daughter's face area is damaged due to the loss of packets in the video sequence. On the other hand, the benefit of MUSC ADP Sub profile is evident in Table 4, where it provides QoS support for a session by adapting it to the current wireless conditions.

In addition to the PSNR, the SSIM of the video sequence was evaluated by using the MSU Video Quality Measurement Tool 1.52 [48]. The SSIM achieves a deservingly approximation of subjective quality assessment methods. Table 5 summarizes the SSIM results obtained in R1 in the foreign network when n_adp_loss and ADP_Sub profiles are used.

The results show that on average the ADP_Sub profile aims to keep the session with an excellent quality level (minimal video distortion, where the average SSIM is of 0.99), while the $n$ adp_loss profile introduces a distortion of $45 \%$ in the video sequence. In the worst scenario, the maximal distortion in the foreign network when the ADP_Sub and $n$ adp_loss profiles are configured is of 0.93 and 0.52 , respectively.

Table 5 SSIM measured in R1 in the foreign network when n_adp_loss and ADP_Sub profiles are configured

\begin{tabular}{lcc}
\hline SSIM & n_adp_loss & ADP_Sub \\
\hline Max & 0.79 & 1.0 \\
Min & 0.52 & 0.93 \\
Avg & 0.65 & 0.99 \\
\hline
\end{tabular}

\subsection{Prototype}

In order to confirm the results obtained by MUSC QoS control solution in the simulation environment and to demonstrate the efficiency of MUSC concerning internetwork adaptation in an experimental QoS-aware mobile network, an adequate test-bed was configured. The IPv4 test-bed is composed by three access networks, one source placed in one access network, one mobile receiver connected to another access network and five routers with MUSC and MIRA agents as illustrated in Fig. 11.

All devices are Pentium IV $1.7 \mathrm{GHz}$ and use a Linux Fedora 2.6.16 kernel. The core routers are equipped with $10 \mathrm{Mb} / \mathrm{s}$ PCI network devices, while the access routers support also a PCI wireless card $802.11 \mathrm{~b} / \mathrm{g}$ with an external antenna and a transmission rate of $11 \mathrm{Mb} / \mathrm{s}$. The source and the receiver are equipped with $11 \mathrm{Mb} / \mathrm{s}$ PCI card IEEE $802.11 \mathrm{~b} / \mathrm{g}$. The Distributed Internet Traffic Generator (DITG) is responsible to send and to receive flows of the session and the mobility is controlled by MIPv4 bidirectional tunnelling. Since inter-network adaptation is the

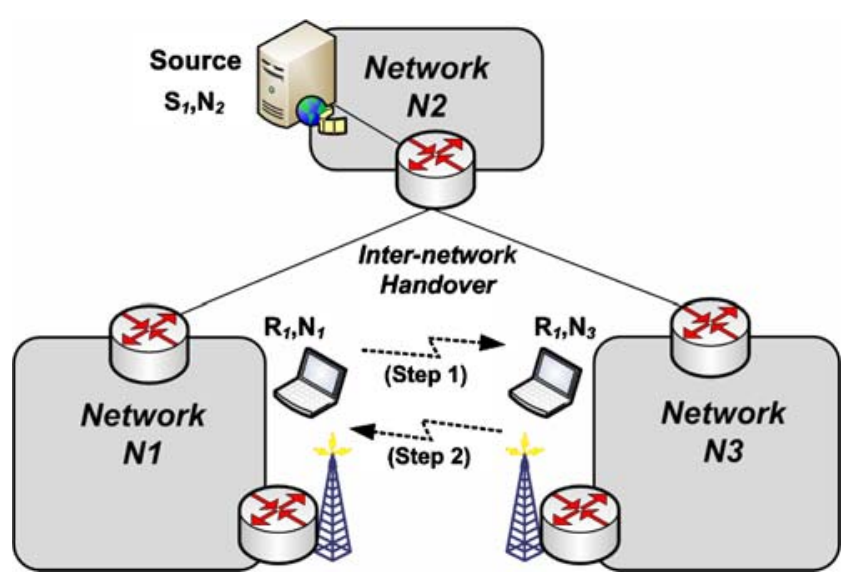

Figure 11 Topology used in the experimental evaluation 
Figure 12 Average throughput and latency for ADP_Drop, $\mathrm{ADP} \mathrm{Hyb}$ and ADP Sub profiles

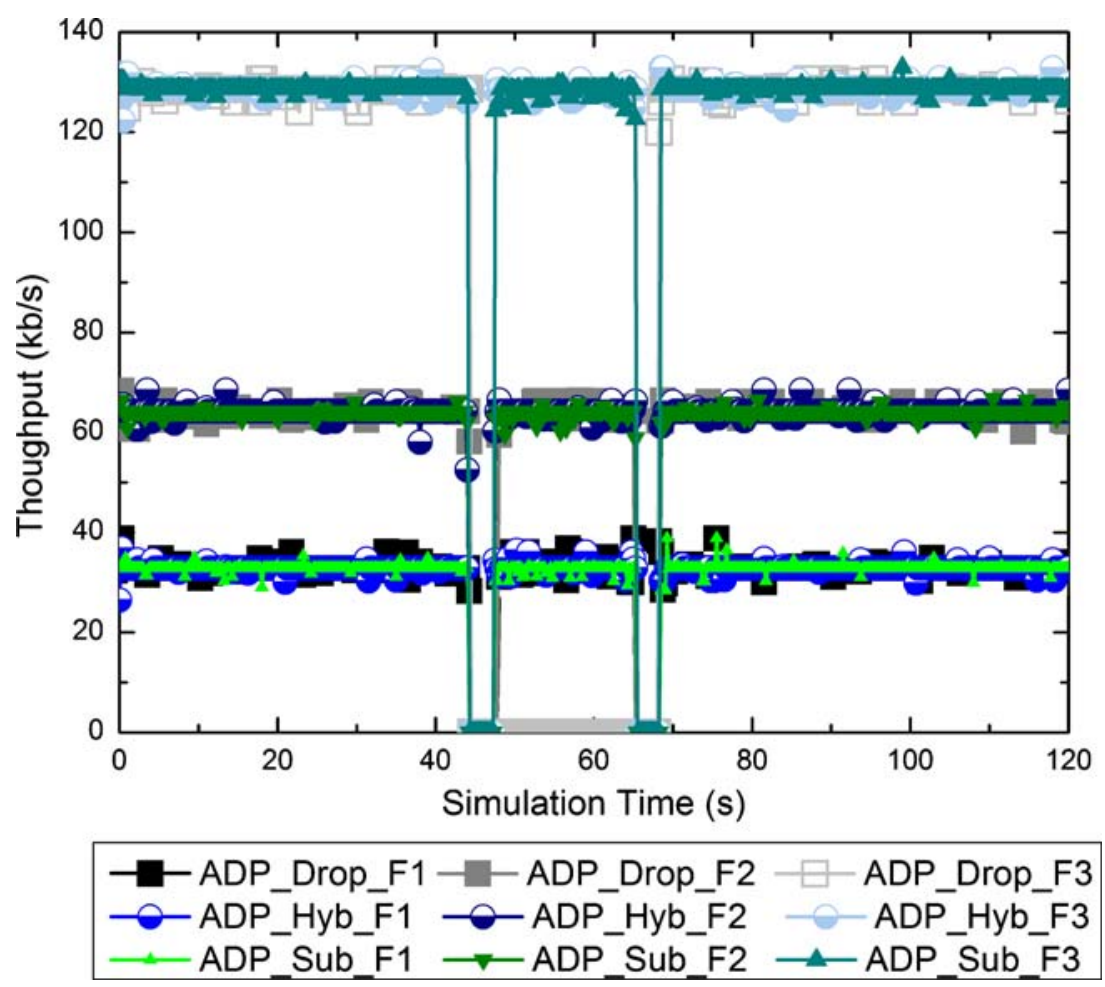

focus of this evaluation, the bandwidth required for the session exceeds the amount of resources assigned to the preferred class in the inter-network link from the access network hosting the source and the receiver's foreign network. Each experiment was repeated ten times, in which the receiver moves to the foreign network $45 \mathrm{~s}$ after its subscription and returns to the home network $65 \mathrm{~s}$ latter. Figure 12 depicts the average throughput measured in the receiver when the ADP_Drop, ADP_Hyb and ADP_Sub profiles are used.

The results confirm the MUSC efficiency in a real scenario and show that MUSC assures the session full rate when ADP_Hyb and ADP_Sub are configured, by using resources available in other service classes. When the profile ADP_Drop is configured, MUSC controls the session quality level and keeps it with an acceptable level, because only the less important flow of the session is affected in the foreign network (F1 and F2 have an average rate of approximately 32 and $64 \mathrm{~kb} / \mathrm{s}$, respectively). The available bandwidth in the home network can accommodate the session full rate in the preferred class. The throughput is zero during the movement of the receiver to the foreign network and during its return to the home network. The standard deviation of the handover latency is $\sim 0.8 \mathrm{~s}$ and the $95 \%$ confidence interval is $\sim 0.51 \mathrm{~s}$.

As explained before, the full rate of ongoing sessions is assured by the ADP Hyb and ADP Sub profiles, while the RTT is degraded. The results obtained for the RTT delay in home and foreign access-networks with all profiles is presented in Table 6. The RTT delay metric was chosen to simplify the experiment, since it does not require time synchronization between the network devices nor extra equipment.

On average, the RTT in the home network, where the session is mapped into the preferred class, is of $1.98 \mathrm{~ms}$. In the foreign network, the average RTT is increased, as shown in the simulation evaluation, due to the encapsulation/decapsulation of packets inherent to the MIP bidirectional tunnelling approach. In addition to the time consumed by the QoS-aware tunnels, the session adaptation to a service class that offers different delay tolerance also influences the RTT. The mobile receiver needs to wait, on average, 3.45, 3.67 and $3.81 \mathrm{~ms}$ when the ADP_Drop, ADP_Hyb and ADP_Sub profiles are used, respectively.

Table 6 RTT delay in home and foreign access-networks for all profiles

\begin{tabular}{lccc}
\hline RTT Delay & $\begin{array}{l}\text { ADP_Drop } \\
(\mathrm{ms})\end{array}$ & $\begin{array}{l}\text { ADP_Hyb } \\
(\mathrm{ms})\end{array}$ & $\begin{array}{l}\text { ADP_Sub } \\
(\mathrm{ms})\end{array}$ \\
\hline Home-Max & 2.18 & 2.21 & 2.10 \\
Home-Min & 1.73 & 1.78 & 1.80 \\
Home-Avg & 2.01 & 2.02 & 2.00 \\
Foreign-Max & 3.45 & 3.67 & 3.81 \\
Foreign-Min & 3.11 & 3.39 & 3.43 \\
Foreign-Avg & 3.45 & 3.99 & 4.22 \\
\hline
\end{tabular}




\section{Conclusion and future work}

This article introduces the MUSC proposal, which provides QoS mapping and adaptation for multi-user sessions over heterogeneous mobile networks. MUSC controls the session quality level for fixed and mobile users independently of the QoS model, service classes and current network conditions on the path from the sender to the receivers. MUSC does not require changes on mobile devices and session coding, which decreases the complexity level of mapping and adaptation mechanisms and avoids the time required by application level re-coding. Even though MUSC was analysed with MIP bi-directional tunnelling and MIRA, it has interfaces, allowing operators to use any other resource allocation controller or mobility scheme of their choice, such as mobility based on PIM-SSM remote subscription.

The simulation reveals that MUSC introduces low latency in handover times to control the session quality level on new paths. It represents on average $0.1 \%$ of the delay spent in all handovers to configure QoS-aware distribution trees for scalable multi-user sessions. In what concerns the performance of the adaptation function, it was shown, for instance, that a controlled degradation of part of already established scalable sessions allows a significant decrease of the session blocking, while keeping all sessions with acceptable quality. Simulation results show that using a selective dropping profile of $25 \%$ and $50 \%$, MUSC was able to accept $85 \%$ and $100 \%$ of scalable multi-user sessions, respectively. Using the adaptation drop profile with a selective dropping limit of $0 \%$, only $50 \%$ of sessions were admitted. However, the average throughput when the selective limit is of $0 \%$ decreases to $89 \%$, while it is reduced to $72 \%$ when the limit is of $25 \%$. In addition, the average throughput of when the system is configured with an aggressive selective limit of $50 \%$ is $67 \%$. Moreover, simulation shows that in a period of congestion of the preferred class, MUSC also keeps ongoing non-scalable sessions with excellent quality level experience.

The results obtained during the simulation concerning inter-network handover are confirmed in an experimental environment. The prototype experiment presents the MUSC efficiency to control the quality level of an ongoing scalable session, in which the results are similar to the ones related in the simulation experiment. The variation of the average throughput measured in the moving receiver (in home and foreign networks), depends on the used QoS adaptation profile. The ADP Hyb and ADP Sub aims to keep the session full rate, while the ADP Drop decreases the session rate in $57 \%$. Nevertheless, the lower throughput imposed by the ADP Drop scheme still provides an acceptable quality level to the session, since flows start to be dropped by the less significant ones. The session RTT is higher in the foreign network due to the creation of MIP tunnels and it is also influenced by the QoS profile method used in the system. The ADP_Sub profile is expected to introduce the highest RTT. The measurements made in the prototype when the receiver moves from its home to the foreign network provides some hints about the most efficient of the tested adaptation schemes. The measured combined performance of ADP Drop, ADP Hyb, and ADP Sub methods was of $(57 \%, 174 \%),(99.5 \%, 185 \%)$, and $(99.2 \%, 192 \%)$, respectively, being the parameters related to the average throughput (first) and RTT (second) variations. As an example of the session quality level in the foreign network, the session full rate is decreased in $57 \%$ (only the two most important flows are accepted), while the RTT delay is increased in $174 \%$.

As future work, heuristics for the combination of all adaptation profiles according to historical data and traffic patterns in large scale networks will be investigated. Further experiments to measure the impact of MUSC from the user's point of view, by analysing other subjective and objective methods will be performed. Regarding the MUSC QoS control behaviour in wireless infrastructures, MUSC will also be investigated in the control of quality level of sessions offered and accessed by devices placed in sensor networks.

Acknowledgment This work was done at the Laboratory of Communications and Telematics of the Faculty of Science and Technology of the University of Coimbra. It is supported by DoCoMo Euro-labs, by the Portuguese Ministry of Science, Technology and High Education, and by European Union FEDER - POSI (projects Q3M and SAPRA).

\section{References}

1. Shanableh T, Ghanabari M (2005) Multilayer transcoding with format portability for multicasting of single-layered video. IEEE Trans Multimedia 7, February

2. Babiarz J,Chan K, Baker F (2006) Configuration guidelines for DiffServ service classes. IETF RFC 4594, August

3. Johnson D, Perkins C, Arkko J (2004) Mobility Support in IPv6. IETF RFC 3775, June

4. Rosenberg J et al (2002) SIP: Session initiation protocol. IETF RFC 3261, June

5. Zhou L, Sun Y (2006) An analysis of multicast support for mobile hosts using mobile IPv6. In: Proc. of IEEE International Conference on Wireless Communications, Networking and Mobile Computing, Wuhan, China, September

6. Cerqueira E et al (2007) QoS Mapping and adaptation in next generation networks. In Proceedings of IEEE International Symposium on Applications and the Internet Workshop, Hiroshima, Japan, January

7. Cerqueira E et al (2007) QoS mapping and adaptation control for multi-user sessions over heterogeneous wireless networks. In: Proceedings of ACM International Mobile Multimedia Communications Conference, Nafpaktos, Greece, August

8. Mammeri Z (2005) Approach for end-to-end QoS mapping and handling. In: Proceedings of IEEE/IFIP Wireless and Optical Communications Networks, Dubai, UAE, March 
9. Ben Ali R et al (2005) UMTS-to-IP QoS mapping for voice and video telephony service. IEEE Netw 19:26-32

10. Chen J et al (2005) An integrated QoS control architecture for IEEE 802.16 broadband wireless access systems. In: Proceedings of IEEE Global Telecommunications Conference, St. Louis, MO, USA, November

11. El-Gendy $\mathrm{M}$ et al (2004) Paving the first mile for QoS-dependent applications and appliances. In: Proceedings of IEEE International Workshop on QoS, Montreal, Canada, June

12. Ruy $M$ et al (2006) QoS class mapping over heterogeneous networks using Application Service Map. In: Proceedings of IEEE International Conference on Networking, International Conference on Systems and International Conference on Mobile Communications and Learning Technologies, Washington, USA, April

13. Schantz R et al (2006) Controlling quality-of-service in distributed real-time and embedded systems via adaptive middleware: experiences with auto-adaptive and reconfigurable systems. Softw Pract Exp 36:1189-1208

14. Rajan G et al (2006) Policy based QoS architecture in MUSE. In: Proceedings of IEEE Conference on Computer Communications, Barcelona, Spain, April

15. Johansen S, Kim A, Perkis A (2007) Quality incentive assisted congestion control for receiver-driven multicast. In Proceedings of IEEE International Conference on Communications, Glasgow, UK, June

16. Kim J et al (2006) Efficient video transcoding technique for QoSbased home gateway service. IEEE Trans Consum Electron 52 (1):129-137

17. Zhu P, Zeng W, Li C (2007) Joint design of source rate control and QoS-aware congestion control for video streaming over the internet. IEEE Trans Multimedia 9(2):366-376

18. Cerqueira E et al (2006) A unifying architecture for publishsubscribe services in the next generation IP networks. In: Proceedings of IEEE Global Telecommunications Conference, San Francisco, USA, November

19. Cerqueira E et al (2007) Q3M: QoS architecture for multi-user mobile multimedia sessions in 4G systems. In: Proceedings of IFIP/IEEE International Conference on Management of Multimedia and Mobile Networks and Service, San Jose, USA, October

20. Cerqueira E et al (2006) Multi-user session control in the next generation wireless system. In: Proceedings of ACM International Workshop on Mobility Management and Wireless Access, Torremolinos, Spain, October

21. Hancook R et al (2005) Next step in signaling (NSIS): framework. IETF RFC 4080, June

22. Ash J et al (2008) QoS NSLP QSPEC template. IETF internet draft, February

23. Handley M, Jacobson V, Perkins C (2006) SDP: session description protocol. IETF RFC 4566, July

24. Bhattacharyya S (2003) An overview of source-specific multicast (SSM). IETF RFC 3569, July

25. Moy J (1998) OSPF version 2. IETF RFC 2328, April

26. Rekhter Y, Li T, Hare S (2006) A border gateway protocol 4 (BGP-4). IETF RFC 4271, January

27. Veloso L et al (2007) Seamless mobility of users for media distribution services. In: Proceedings of IEEE International Performance Computing and Communications Conference, New Orleans, USA, April

28. Chaskar H (2003) Requirements of a Quality of Service (QoS) Solution for Mobile IP. IETF RFC 3583, September
29. Veloso L et al (2007) Seamless Mobility of Users with QoS and Connectivity Support. In: Proceedings of IEEE Conference on Wireless and Mobile Computing, Networking and Communications, New York, USA, October

30. Neto A et al (2008) An integrated approach to control the quality level of multi-user sessions. In: Proceedings of ACM International Workshop on the Evaluation of Quality of Service through Simulation in the Future Internet, Marseille, France, March

31. Neto A et al (2007) A resource reservation protocol supporting QoS-aware multicast trees for next generation networks. In: Proceedings of IEEE Symposium on Computers and Communications, Aveiro, Portugal, July

32. Ash J et al (2008) QoS NSLP QSPEC template. IETF internet draft, February

33. Mendes P, Andres-Colas J, Pinho C (2005) Information model for the specification of QoS agreements among ambient networks. In Proceedings of IEEE Symposium on Personal Indoor and Mobile Radio Communications, Berlin, Germany, September

34. Chen $X$ et al (2006) Supporting QoS in IEEE 802.11e Wireless LANs. IEEE Trans Wireless Commun 5(8):2217-2227

35. Mendes P, Schulzrinne H, Monteiro E (2004) How to increase the efficiency of receiver-driven adaptive mechanisms in a next generation of IP networks. Comput Commun 27:345-354

36. Wang X, Schulzrinne H (2001) Pricing network resources for adaptive applications in a differentiated services network. In: Proceedings of IEEE Conference in Computer Communications, Anchorage, USA, April

37. Sanda T et al (2008) Applicability statement of NSIS protocols in mobile environments. IETF internet draft, February

38. Di Z, Mouftah H (2001) Performance evaluation of per-hop forwarding behaviours in the DiffServ Internet. In: Proceedings of IEEE Symposium on Computers and Communications, AntibesJuan les Pins, France, July

39. Rose K, Regunathan S (2001) Toward optimality in scalable predictive coding. IEEE Trans Image Process 7:965-976

40. Video Traces Research Group (2007) YUV 4:2:0 video sequences. Arizona State University, September. Available at: http://trace.eas. asu.edu/yuv/qcif.html

41. Ke C et al (2006) A novel realistic simulation tool for video transmission over wireless network. In: Proceedings of IEEE International Conference on Sensor Networks, Ubiquitous, and Trustworthy Computing, Taichung, Taiwan, June

42. Kimura J et al (1999) Perceived quality and bandwidth characterization of layered MPEG-2 video coding. In: Proceedings of SPIE International Symposium, USA, September

43. ITU-R Recommendation BT.500-10 (2000) Methodology for subjective assessment of the quality of television picture.

44. ANSI T1.801.03-1996 (1996) Digital transport of one-way video signals - parameters for objective performance assessment

45. ISO-IEC/JTC1/SC297WG11 (1996) Evaluation methods and procedures for July MPEG-4 tests

46. Wang $\mathrm{Z}$ et al (2004) Image quality assessment: from error visibility to structural similarity. IEEE Trans Image Process 13(4):600-612

47. Gvozden G, Gosta M, Grgic S (2007) Comparison of H.264/AVC and MPEG-4 ASP coding techniques designed for mobile applications using objective quality assessment methods. In: Proceedings of IEEE International Symposium ELMAR-2007 focused on Mobile Multimedia, Zadar, Croatia, September

48. Vatolin D, Moskvin A, Petrov O (2008) MSU video quality measurement tool 1.52. Available at: http://compression.ru/video/ quality_measure/video_measurement_tool_en.html, accessed in March 